\title{
A Survey On Automated Methods Used For WBC Classification
}

\author{
Chandni Patodia ${ }^{1}$, Nibhasya $\mathrm{G}^{2}$, Prof. Chandraprabha $\mathrm{R}^{3}$ \\ ${ }^{1,2}$ Student in Department of Electronics and Communication \\ BMS Institute of Technology and Management, Bangalore, Karnataka, India \\ ${ }^{3}$ Assistant Professor in Department of Electronics and Communication \\ BMS Institute of Technology and Management, Bangalore, Karnataka, India
}

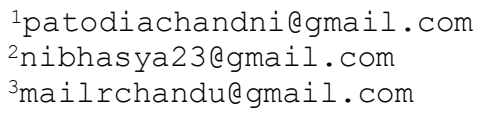

\begin{abstract}
The conventional process of the well-known Complete Blood Count test or abbreviated as CBC test has provided pathologists and hematologists with information for diagnosing blood related diseases. This manual method has proved to be tedious, bias and inaccurate. Thus, automated methods for blood cell detection and counting have been sought out to improve the accuracy for better diagnosis. These automated methods lead to early detection of blood related diseases such as anemia, leukemia, lymphoma and other infections in the immune system. To find an alternative to the manual methods, this paper provides a comparative table of 12 papers which were thoroughly surveyed to draw conclusions from their automated methodologies used and final outcomes. This paper can be useful in the future for knowing which methods gave the best and highest accuracies and further advancements can be done based on the inferences made from the literature survey done in this paper. It's just a bird's overview of the diagnosis of blood cells and their applications in the medical field.
\end{abstract}

Keywords - Automated Methods, Blood Diseases, Classification, Comparative study, Convolutional Neural Networks (CNN), White Blood Cells (WBCs)

\section{INTRODUCTION}

Blood is considered as a tissue and is generally thick as it is composed of a variety of cells, each having a unique task. Major forms of blood cells include; White blood cells (leukocytes), Red blood cells (erythrocytes), Platelets (thrombocytes).

Checking the quantity or count of white blood cells is a part of the novel complete blood cell (CBC) test. It is usually used to look for various diseases that might be present in the body. Pathological image analysis for blood cell recognition has a remarkable impact on the diagnosis process in the medical field. The computer compares the operations and detects the object regardless of where it is located in the image. The diseases are determined based on the number of blood cells which were infected. For example, many diseases like cancer, leukemia, malnutrition, etc. can be diagnosed based on the classification and quantity of red and white blood cells. White Blood Cells are majorly responsible for fighting against any kind of infection, and increased white blood cell count may be caused by alternative problems including inflammation and stress.

A manual pathological review is incredibly time consuming and tedious, and there's great interest in developing image analysis systems that may assist pathologists by automating routine tasks and performing speculative segmentation and classification of images. This paper is an attempt to summarize the literature survey conducted with 12 papers based on White Blood Cells (WBCs) and its applications. It sheds light at each paper's methodology, authors and final outcome. The larger goal is to develop a system that can speculatively classify cells into respective categories with pathological significance to assist pathologists in their daily workflow. If the algorithm can identify the correct structures and classify them accurately, it would be possible for the computer to make a diagnosis or compute some preliminary diagnostic score. Such a metric would be very fast, have a large baseline of objectivity since it can thoroughly examine all the available data and be easily scalable. This would be a powerful addition to current clinical practice.

WBCs are available in many different shapes and sizes. Some of them are mononuclear and some are polynuclear. WBCs are produced from the bone marrow and located within the blood and vascular system. There are five types of WBCs namely Neutrophil, Eosinophil, Basophil, Monocyte, Lymphocyte. Depending upon which WBCs are missing, the patient is in danger of various varieties of infection.

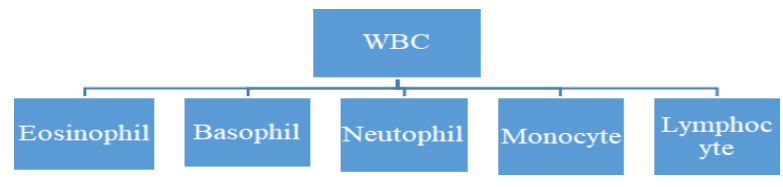

Fig. 1.WBC Classification 


\section{International Journal of Engineering Technology and Management Sciences[IJETVIS]}

Website: ijetms.in Issue:5, Volume No.4, September-2020 DOI: 10.46647/ijetms.2020.v04i05.006

\section{LITERATURE SURVEY}

\section{A. Classification of WBC for Blood Cancer Diagnosis using Deep Convolutional Neural Networks}

Author: Dhvani Kansara, Shaunak Sompura, Saifil Momin, Mitchell D‘silva

Methodology: In this paper, for the diagnosis of blood related diseases, classifying WBCs into their subtypesEosinophils, Neutrophils, Monocytes and Lymphocytes from blood samples images is implemented using an automated method. This provided a time efficient and an accurate diagnostic system. Non-linearity was established using Rectifier Activation function. To impede the feature map from shrinkage, padding was done by appending zeros around the input. Addition of a pooling layer in between the convolution layers was common to decrease the number of computations by reducing the dimensionality. There were various pooling functions like, max pooling, average pooling and sum pooling which could be used. The following layers were accountable for the categorization of WBCs. This was composed of fully connected layers which accepted data in single dimensionality (1D). Thus, 3D data was converted to $1 \mathrm{D}$ by application of the flattened layer to the output of feature extraction. The fully connected layer was followed by the dense layer which formed the hidden layer of neurons where back propagation was applied, followed by the SoftMax layer which provided a binary output, which denoted if an input image belonged to a certain class or not.

Final Outcome: The model used 30 epochs for improving accuracy. Deep learning proved outstandingly better than conventional approaches, as it was able to recognize the minute complexities in the image to categorize them precisely. It also provided improved feature extraction, which learnt about complex non-linear relations between independent and dependent variables. The execution was a scalable approach. $83 \%$ was obtained as precision, which means that $83 \%$ of the time the results were correctly obtained. A recall average of $78 \%$ showed that the model could rightly classify 78 out of 100 cases. They obtained an F1-score of $78 \%$, which conveys the balance between precision and recall [1].

\section{B. Combining Neural Network Models for Blood Cell Classification}

Author: Indraneel Ghosh, Siddhant Kundu

Methodology: The objective of the study was to evaluate the efficiency of a multi-layer neural network models built by combining Convolutional Neural Network (CNN) and Recurrent Neural Network (RNN) for solving the problem of classifying of different kind of White Blood Cells. This paper consists of five parts - Input Layer, CNN Layer, RNN Layer, Merge Layer and Output Layer. The dataset images were augmented by rotation, reflection about the horizontal axis and shifting both horizontally and vertically. The CNN and RNN models were trained simultaneously. The CNN layer in this model has 4 layers. Each layer uses a $3 \times 3$ kernel. The RNN layer consists of LSTMs that learn to detect recurring features in the images. The images were converted to grayscale before being passed to the RNN. Finally, the output layer has 128 cells and uses SoftMax function to maximize the classification efficiency.

Final Outcome: It can be observed that, the CNN-RNN hybrid architecture offers better results than the CNN-only network - for the four-way classification problem, approximately $8 \%$ of the cases were misclassified by the CNN-only solution and were classified correctly by the CNN-RNN combination, assuming that the hybrid network does not misclassify the items that are correctly classified by the CNN-only network. For the two-way classification problem, approximately $6 \%$ of the misclassified cases were precisely classified by the hybrid network. With the usage of the optimized DNN version of the LSTM layers, the training time was roughly the same as with the pure CNN network (approximately 13 seconds per epoch for CNN only, approximately 17 seconds per epoch on $\mathrm{CNN}+\mathrm{RNN}$ ). Thus, the usage of an RNN alongside a CNN leads to an improved performance. It was noted that, it is difficult to detect small objects due to the limited discriminativeness of the global visual features. It only predicts a label, not a segmentation box [2].

\section{Classification of White Blood Cells by Deep Learning Methods for Diagnosing Disease}

Author: Muhammed Yildirim, Ahmet Çinar Methodology: In this paper, Convolutional Neural Network (CNN) was implemented to distinguish between several types of white blood cells. The CNN model was integrated with architectures like DenseNet201, AlexNet, GoogleNet, ResNet50; and used Kaggle's dataset for training. Then, Gaussian and median filters were applied separately to the image dataset. The four networks classified new images. Original data was classified with the four architectures, after which confusion matrices and accuracy values were attained. Then, filters (Median and Gaussian) were applied to the dataset and new data was obtained which were then reclassified and accuracy value was obtained, and confusion matrices were plotted for the same.

Final Outcome: In this paper, results procured for the data which had filters applied showed better results than original data. It was easier to draw inferences about diseases, once blood cells had been classified. The highest accuracy of $79.27 \%$ was attained by AlexNet. Similarly, Resnet50 attained $78.74 \%$, DenseNet201 obtained $77.88 \%$ and GoogleNet with $62.93 \%$ followed. On applying the median filter, AlexNet achieved the highest accuracy rate of $80.77 \%$, which was followed by DenseNet201 with $77.67 \%$, ResNet50 with $74.89 \%$ and GoogleNet with 


\section{International Journal of Engineering Technology and Management Sciences[IJETMS]}

Website: ijetms.in Issue:5, Volume No.4, September-2020 DOI: 10.46647/ijetms.2020.v04i05.006

69.44\%. After applying Gaussian filter, DenseNet201 architecture attained the highest accuracy of $83.44 \%$, followed by AlexNet with $82.16 \%$, ResNet50 with $80.02 \%$ and GoogleNet with 75.21\% [3].

\section{White blood cells classification with deep convolutional neural networks}

Authors: Ming Jiang, Liu Cheng, Feiwei Qin, Lian Du, Min Zhang

Methodology: In this paper, a new convolution neural network model is constructed named WBCNet. This model is capable of extracting all features of microscopic WBC image by using a combination of residual convolution architecture, batch normalization algorithm, and improved activation function. The WBCNet consists of 33 layers, whose speed is better as compared to the conventional $\mathrm{CNN}$ model during the training phase. Category of white blood cell can also be quickly identified. During the training phase, data enters the model from the input layer, which then moves ahead in the WBCNet. The model further produces a predictive output for every image. They use the Caffe framework to train model and CUDA 8.0 to speed up the implementation.

Final Outcome: An epoch of 800 cycles are back propagated. WBCNet achieved highest accuracy rate when trained with $110-130$ epochs (i.e. 88,000 to 10400 iteration). Top-1 accuracy rate of $77.65 \%$, top-5 accuracy rate of $98.65 \%$ was attained. For testing generalization capabilities of WBCNet, 20400 images were chosen additionally that were not trained. The generalization ability of the model was better, and the accuracy rate of approximately 50 test datasets was about $83 \%$. In the additional 20400 images, 16932 images were classified correctly and 3468 were wrongly classified. WBCNet's rate of convergence was faster, the training data fits better, and the accuracy was higher. Ultimately, the experiment concluded that WBCNet was overall better than the customary models [4].

\section{E. Automated AML Detection from Complete Blood Smear Image Using KNN Classifier}

Author: Nayana B. Sen, Mercy Mathew

Methodology: This work presented a simple technique which automatically detects Acute Myelogenous Leukemia (AML) which is a sub-category of acute leukemia. The proposed method was simple, and it differs from other papers by the use of complete blood smear image for classification. The system will be analyzed features from microscopic images and examined changes in texture, geometry, color, Hausdorff Dimension (HD) and statistical characteristics. The segmentation of nucleus from blood smear image was performed using color-based algorithm which was more efficient than existing algorithm. The classification process was done using KNN classifier. The system was exercised to blood smear images comprising of multiple nuclei. Two features had been used for classifying: cell energy and Hausdorff dimension (HD). The segmentation and classification work on a step-by-step procedure. The proposed system consisted of the following stages: Image Acquisition, Segmentation, Feature Extraction and Classification.

Final Outcome: The system presented performed automated processing, which included color correlation, segmentation of the cells with nucleus, classification and feature extraction. The HD was found on the basis of box counting. The number of white blood cells in the images of cancerous case was more than that of the normal case. There was a comparable difference in HD. That is why, the $\mathrm{HD}$ proved to be an important feature in the proposed system. Classification of a specimen resulted in four possibilities: false positives (FP), when noncancerous cells are identified as cancerous; false negatives (FN), when cancer cells were identified as noncancerous; true negatives (TN), when noncancerous cells are correctly identified; and true positives (TP), when cancer cells are correctly identified [5].

\section{F. White Blood Cells Detection and Classification Based on Regional Convolutional Neural Networks}

Author: Hüseyin Kutlu, Engin Avci, Fatih Özyurt Methodology: The motivation for this study was to use Deep Learning method to escalate the performance of existing blood testing systems. The proposed methodology used Regional Convolutional Neural Networks (R - CNN). In this model, different types of cells within the same image were classified concurrently with a detector. While training CNN was the basis of R-CNN model, architectures like VGG16, AlexNet, ResNet50, GoogleNet have also been tested with transfer and full learning. The method comprised of mainly 5 steps: creation of data set, coordinates and classes of WBC are determined in dataset, separating dataset into test and training data, procurement of WBC detector with transfer learning and assessing it with the test data.

Final Outcome: At the end of the study, the proposed method showed 100\% success in defining WBCs. ResNet50 showed the best performance with transfer learning. Accuracy rates of each WBC type were deliberated as $99.52 \%$, Monocyte as $98.40 \%$, Basophil as 98.48\%, Eosinophil as $96.16 \%$ and Neutrophil as $95.04 \%$. This study was different from the traditional image processing methods because of the success of the classifier and WBC detector against the challenges of the object's partial visibility and overlapping in the image. In future studies, the system ought to be implemented with real-time images by training the system with different medical images and different CNN models. It was noted that, in R$\mathrm{CNN}$ for each region CNN was applied separately so that the training time was almost 84 hours which won't be useful in real-time applications [6]. 


\section{International Journal of Engineering Technology and Management Sciences[IJETMS]}

Website: ijetms.in Issue:5, Volume No.4, September-2020 DOI: 10.46647/ijetms.2020.v04i05.006

\section{G. Counting of WBC Using Digital Microscopic Camera in Image Processing}

Author: Poonam Ahire, Tejaswi Gosavi, Shital Bairagi, Pranali More

Methodology: Various methods involving segmentation of blood cells from microscopic images of blood are discussed in this paper. This data is the means to perform higher level tasks for instance, automatic differential blood counting. For identification and classification of various white blood cells, many geometric features are considered for every segment and a color-based segmentation is used. The obtained results are compared with the manual results obtained from the lab and success of the proposed method is demonstrated here. The most economical and quick solution in this paper is to provide Digital Image processing. Shape analysis was used for WBC segmentation. Segmentation is done using thresholding. After that, the shape of WBCs can be determined by shape analysis procedure. In case of shift approach or the color GVF snake (which gives the boundaries in an image), weak textures are not detectable, so all types of WBCs can't be detected. So, these methods are not used here as a result the segmentation performance is much better.

Final Outcome: Their method of identifying leukocytes can also be carried out on other cells like eosinophil and basophil. The proposed model is of less cost and gives better classification results. Based on only feature set used, they have achieved an accuracy of $92 \%$ to $98 \%$. With one of the other feature sets, it provides $98 \%$ to $99 \%$. Good preprocessing methods can improve the accuracy of the model. [7].

\section{H. Classification of WBC Using Deep Learning for Diagnosing Diseases}

Authors: Riya roy, Swapna Sasi

Methodology: This paper concentrated on detecting harmful cells by classifying WBCs. It contains five phases. Microscopic images from blood are taken. There are two stages. Images are collected and then segmented. The features from these images are extracted by various methods in the model. This is considered as the first stage of their paper. In second stage, the cells are classified with the help of deep learning. The SVM classification method is used for this purpose. They made use of Convolutional Neural Networks. The images utilized here are of the size $512 * 512$. All of them are in JPEG format. MATLAB was used here. The details of the number of nucleus, the cytoplasm and ratio between their areas are given by the structural features.

Final Outcome: The common diseases in various age groups can be diagnosed by the proposed model, and the cells can be classified automatically. The accurate cell size is also given here. Using this they were able to identify the diseases caused by unhealthy WBCs. This paper does not give any specific outcomes. The paper is very generalized regarding the diseases caused due to unusual development/ variety of cells [8].

\section{White Blood Cell Classification and Counting Using Convolutional Neural Network}

Author: Merl James Macawile, Vonn Vincent Quiñones, Alejandro Ballado Jr., Jennifer Dela Cruz, Meo Vincent Caya

Methodology: In this paper, HSV (Hue, Saturation, Value), a saturation component is used along with blob analysis to segment numerous forms of WBCs i.e. neutrophils, lymphocytes, basophils, eosinophils and monocytes from a microscopic image of blood. CNN (Convolutional Neural Network) is integrated to count WBCs in the blood which can generate better results. This paper includes a series of steps employed for classifying the leukocytes from the blood images. The samples are collected from an online source called enjoy pathology and ALL-IDB database with different qualities, to check the adaptability of the process in separating the WBCs from blood. The image resolutions range from 350x236 till $2592 \times 1944$ as they were taken from different magnifications of microscope. An image processing toolbox i.e. MATLAB was utilized to inspect the images. They used a laptop of i7 processor with 16GB RAM. 178 WBC were taken from 21 blood images to evaluate the model and obtain a higher accuracy.

Final Outcome: The WBC which were segmented from the sample blood images are taken as validation images to provide unbiased evaluation. Alexnet, ResNet-101 and GoogLeNet which are the pretrained models of CNN are used here for the classification process. To access the performance of the classification models, specificity, sensitivity, and accuracy are taken into consideration which are the statistical measures. To calculate the statistical measures, the data can be labelled as True Positive (TP), True Negative (TN), False Negative (FN) and False Positive (FP) as per the confusion matrix. Alexnet has the best performance i.e. a good accuracy of all the three models implemented here in classification and counting of the WBCs from 21 sample images. This model obtained a $97.85 \%$ specificity, $89.18 \%$ sensitivity, and an accuracy of $96.63 \%$ [9].

\section{J. Subclass Separation of White Blood Cell Images Using Convolutional Neural Network Models}

Authors: Mesut Togacar, Burhan Ergen, Mehmet Emre Sertkaya

Methodology: This paper focuses on separating WBCs from the blood and their classification using Convolutional Neural Network (CNN), which is a deep learning algorithm. The WBC data set contains a total of 12,435 images. Of which 3,091 are monocytes, 3,120 are eosinophils, 3,102 are lymphocytes, and 3,122 are 
Website: ijetms.in Issue:5, Volume No.4, September-2020 DOI: 10.46647/ijetms.2020.v04i05.006

neutrophils. The resolution of each image in the dataset is $320 \times 240$ pixels and they are of JPEG format. Various architectures like LeNet, AlexNet, and VGG-16 are used for feature extraction. Optimization methods like Stochastic Gradient Descent, Adam, SGD, RMSprop were used in the process of extracting features during classification. Many classifiers like Softmax, DT, QDA, LDA, SVM and KNN are used here. Both MATLAB tool and Python programming are combined in the case of Alexnet used here.

Final Outcome: Of all the convolutional neural network architectures used here, Alexnet provides better accuracy in the WBC subclass separation. Quadratic discriminant analysis classifier provides an outstanding performance in classifying white blood cells. This paper used a number of classifiers like SoftMax (accuracy of 84.47\%), DT, QDA, LDA, SVM and KNN. Among these QDA gave the highest accuracy of $97.78 \%$. The values from the confusion matrix determines the performance of the model. Super pixel method was implemented on the same subtypes of WBCs in the data set to increase the efficiency. So, this can be used in further research studies to improve the success rate. The use of PCA along with CNN model did not provide good results [10].

\section{K. Classification of White Blood Cells with Pattern Net- fused Ensemble of Convolutional Neural Networks (PECNN)}

Authors: Justin L. Wang, Anthony Y. Li, Michelle Huang, Ali K. Ibrahim, Hanqi Zhuang, Ali Muhamed Ali Methodology: Pattern Net-fused Ensemble of Convolutional Neural Networks (PECNN) is created and utilized in this paper for the classification of leukocytes. The architecture proposed depends on a composite method, Pattern Net, where the outputs of randomly produced Convolutional Neural Networks (CNNs) are combined. The proposed algorithm can be adaptable to the data, by depending upon the generated models. So, it has a wide range of applications. The Pattern Net takes only the strengths of all the models and doesn't consider outliers. In this paper, main focus was on data fusion which increases the model performance. This new model has a simple structure and it is inexpensive computationally, compared to deep CNN. Furthermore, this provides a generalized structure which averts overfitting as it can adapt the data. This is motivated by Random Multimodal Deep Learning (RMDL). In this the text file and image classification depend upon the decisions of generated Deep Neural Networks, CNNs and LSTM networks.

Final Outcome: The plan of employing a Pattern Net for model fusion wasn't to compare every model separately. This also doesn't degrade non ideal methods. In this paper, PECNN, an aggregate model, that depends on combining the results appropriately from a group of randomly produced neural network models, was provided for the classification of WBCs. This paper exhibits that PECNN attained better performances with respect to specificity, precision and accuracy. The proposed model has large range of applications due to its flexibility and simplicity, allowing it to be productive in medical and other fields. Furthermore, due to its simple architecture this can be used and modified for other applications [11].

\section{Automatic White Blood Cell Detection and Identification Using Convolutional Neural Network}

Authors: Filip Novoselnik, Ratko Grbić, Irena Galić, Filip Dorić

Methodology: In this paper, with the help of less expensive camera and microscope, the dataset can be acquired. By integrating this with an apt algorithm, identification and detection of WBCs can be done from a blood image. This proposed method consists of two parts. The first part is to detect the WBCs based upon the segmentation process. The second part is to classify the blood cells into respective sub classes. A blood sample is taken and segmented to get WBCs from the blood and these are classified further into 5 sub types. All WBCs can be ascertained from segmentation algorithm. LeNet-5 architecture, a pretrained model of CNN is modified and used in this paper. The input is an RGB image which has a resolution of $300 \times 300$ pixels. This architecture has 3 alternating convolution and 3 pooling layers.

Final Outcome: This proposed model has a high recall of $96.6 \%$ and an accuracy of $90.62 \%$ for segmentation of WBCs. The performance of CNN was determined by 85 images for WBC classification. Out of all the test dataset, 22 images are neutrophils, 18 are monocytes, 22 are lymphocytes and the remaining 20 images contain fragments of blood where WBCs are absent. As dataset is very low, only 3 images of eosinophils were accessible. In the created model, $\mathrm{CNN}$ makes a bad prediction on neutrophils and eosinophils. An accuracy of $81.11 \%$ is attained on the created dataset. Differential blood count can be procured effortlessly based on the results of the proposed method. The main limitation of segmentation method introduced in this paper, is that all the parameters should be manually allocated to the suitable values [12]. 
Website: ijetms.in Issue:5, Volume No.4, August-2020

TABLE I

SUMMARY

\begin{tabular}{|c|c|c|c|c|c|}
\hline $\begin{array}{l}\text { Serial } \\
\text { No }\end{array}$ & Title & Author & Methodology & Final Outcome & Limitations \\
\hline 1 & $\begin{array}{l}\text { Classification } \\
\text { of WBC for } \\
\text { Blood Cancer } \\
\text { Diagnosis } \\
\text { using Deep } \\
\text { Convolutional } \\
\text { Neural } \\
\text { Networks }\end{array}$ & $\begin{array}{l}\text { Dhvani Kansara, } \\
\text { Shaunak Sompura, } \\
\text { Saifil Momin, } \\
\text { Mitchell D`silva }\end{array}$ & $\begin{array}{ll}\text { - } & \text { Rectifier Activation } \\
\text { function is used to } \\
\text { introduce non-linearity } \\
\text { - } \text { Used SoftMax function }\end{array}$ & $\begin{array}{l}\text { - } \quad \text { Binary output } \\
\text { - } 30 \text { epochs are used } \\
\text { - } 83 \% \text { accuracy } \\
\text { - } 78 \% \text { of both recall } \\
\quad \text { average and F1-scor }\end{array}$ & $\begin{array}{l}\text { - Only } 30 \text { epochs are } \\
\text { used } \\
\text { - Less than } 85 \% \\
\text { efficiency }\end{array}$ \\
\hline 2 & $\begin{array}{l}\text { Combining } \\
\text { Neural } \\
\text { Network } \\
\text { Models for } \\
\text { Blood Cell } \\
\text { Classification }\end{array}$ & $\begin{array}{l}\text { Indraneel Ghosh, } \\
\text { Siddhant Kundu }\end{array}$ & 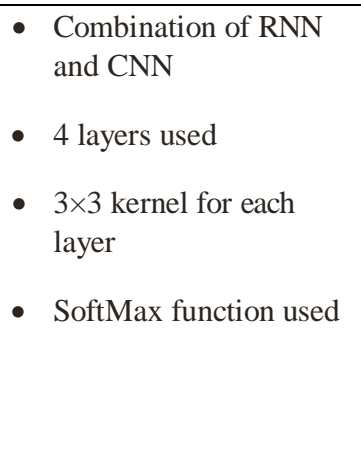 & $\begin{array}{l}\text { - CNN RNN hybrid } \\
\text { offers better result } \\
\text { - } \\
\text { Improved performance }\end{array}$ & $\begin{array}{l}\text { - The hybrid uses } 17 \\
\text { seconds per epoch } \\
\text { whereas CNN uses } 13 \\
\text { seconds per epoch } \\
\text { - Difficult to detect } \\
\text { small objects due to } \\
\text { limited } \\
\text { discriminativeness of } \\
\text { the global visual } \\
\text { features } \\
\text { Only predicts a label, } \\
\text { not a segmentation box }\end{array}$ \\
\hline 3 & $\begin{array}{l}\text { Classification } \\
\text { of White } \\
\text { Blood Cells } \\
\text { by Deep } \\
\text { Learning } \\
\text { Methods for } \\
\text { Diagnosing } \\
\text { Disease }\end{array}$ & $\begin{array}{l}\text { Muhammed } \\
\text { Yildirim, Ahmet } \\
\text { Çinar }\end{array}$ & $\begin{array}{l}\text { - Alexnet, Densenet201, } \\
\text { GoogleNet and } \\
\text { Resnet50 were } \\
\text { integrated in the CNN } \\
\text { model } \\
\text { - Gaussian and Median } \\
\text { filters used }\end{array}$ & $\begin{array}{l}\text { - AlexNet had highest } \\
\text { accuracy of } 79.27 \% \\
\text { (without filter) } \\
\text { - DenseNet gave highest } \\
\text { accuracy of } 83.44 \% \\
\text { (with Gaussian filter) } \\
\text { - AlexNet gave highest } \\
\text { accuracy of 80.77\% } \\
\text { (with median filter) }\end{array}$ & $\begin{array}{l}\text { - The results obtained } \\
\text { were all less than } 85 \% \\
\text { accuracy }\end{array}$ \\
\hline 4 & $\begin{array}{l}\text { White blood } \\
\text { cells } \\
\text { classification } \\
\text { with deep } \\
\text { convolutional } \\
\text { neural } \\
\text { networks }\end{array}$ & $\begin{array}{l}\text { Ming Jiang, Liu } \\
\text { Cheng, Feiwei } \\
\text { Qin, Lian Du, Min } \\
\text { Zhang }\end{array}$ & $\begin{array}{l}\text { - Created own net called } \\
\text { WBCNet } \\
\text { - } 33 \text { layers of network } \\
\text { architecture } \\
\text { - Use the Caffe } \\
\text { framework and CUDA } \\
8.0 \text { to speed up } \\
\text { implementation }\end{array}$ & $\begin{array}{l}\text { - } 110-130 \text { epochs are } \\
\text { used } \\
\text { - Accuracy rates like } \\
77.65 \% \text { and } 98.65 \% \\
\text { were obtained } \\
\text { - The achieved rate of } \\
\text { convergence was faster } \\
\text { and the training data } \\
\text { fits better with higher } \\
\text { accuracy }\end{array}$ & $\begin{array}{l}\text { - Approximately } 20 \% \text { of } \\
\text { the images were } \\
\text { wrongly classified }\end{array}$ \\
\hline 5 & $\begin{array}{l}\text { Automated } \\
\text { AML } \\
\text { Detection } \\
\text { from } \\
\text { Complete } \\
\text { Blood Smear } \\
\text { Image Using } \\
\text { KNN } \\
\text { Classifier }\end{array}$ & $\begin{array}{l}\text { Nayana B. Sen, } \\
\text { Mercy Mathew }\end{array}$ & $\begin{array}{l}\text { - Technique } \\
\text { automatically detects } \\
\text { Acute Myelogenous } \\
\text { Leukemia (AML) } \\
\text { - Complete blood smear } \\
\text { image used } \\
\text { - KNN classifier used } \\
\text { - Features used were cell } \\
\text { energy and Hausdorff }\end{array}$ & $\begin{array}{l}\text { - HD found out based on } \\
\text { box counting } \\
\text { - Detection of cancerous } \\
\text { and non-cancerous } \\
\text { cells only }\end{array}$ & $\begin{array}{l}\text { - Only two features were } \\
\text { used for classification }\end{array}$ \\
\hline
\end{tabular}


Website: ijetms.in Issue:5, Volume No.4, August-2020

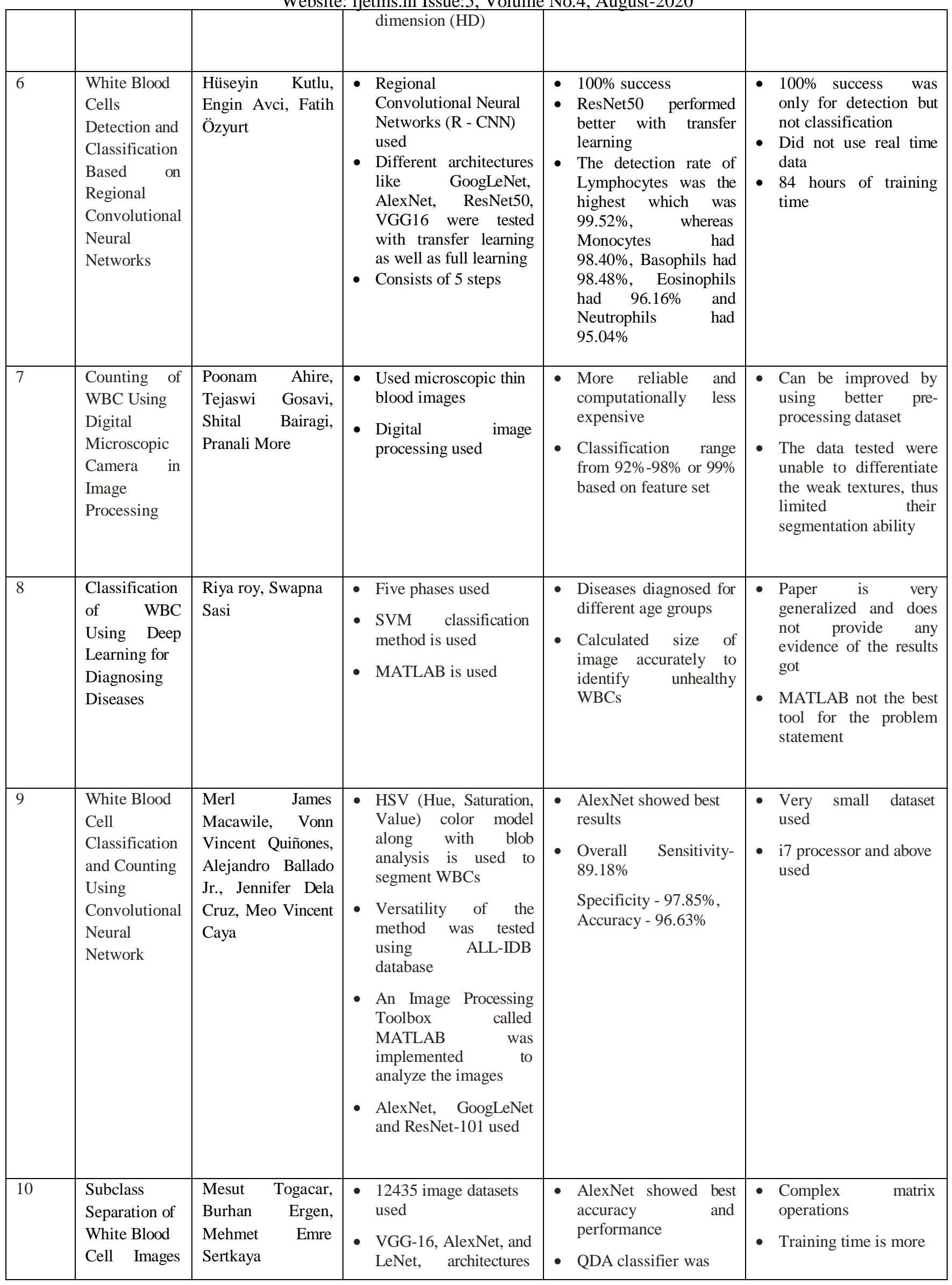




\begin{tabular}{|c|c|c|c|c|c|}
\hline & $\begin{array}{l}\text { Using } \\
\text { Convolutional } \\
\text { Neural } \\
\text { Network } \\
\text { Models }\end{array}$ & & $\begin{array}{l}\text { used } \\
\text { Image processing tool } \\
\text { like MATLAB and } \\
\text { Programming } \\
\text { languages like Python } \\
\text { were used here }\end{array}$ & $\begin{array}{l}\text { better than all the other } \\
\text { classifiers used and } \\
\text { gave accuracy of } \\
97.78 \% \\
\text { - Super pixel method } \\
\text { was used to increase } \\
\text { success rate } \\
\text { CNN model with PCA } \\
\text { didn't give positive } \\
\text { results }\end{array}$ & \\
\hline 11 & $\begin{array}{l}\text { Classification } \\
\text { of White } \\
\text { Blood Cells } \\
\text { with Pattern } \\
\text { Net-fused } \\
\text { Ensemble of } \\
\text { Convolutional } \\
\text { Neural } \\
\text { Networks } \\
\text { (PECNN) }\end{array}$ & $\begin{array}{l}\text { Justin L. Wang, } \\
\text { Anthony Y. Li, } \\
\text { Michelle Huang, } \\
\text { Ali K. Ibrahim, } \\
\text { Hanqi Zhuang, Ali } \\
\text { Muhamed Ali }\end{array}$ & $\begin{array}{l}\text { Used a new method, } \\
\text { Pattern Net-fused } \\
\text { Ensemble } \\
\text { Convolutional Neural } \\
\text { Networks (PECNN) } \\
\text { - Motivated by Random } \\
\text { Multimodal Deep } \\
\text { Learning (RMDL) }\end{array}$ & $\begin{array}{l}\text { - This model is simple } \\
\text { and inexpensive in } \\
\text { comparative to deep } \\
\text { CNN } \\
\text { - Architecture accessible } \\
\text { audience due to light } \\
\text { weight }\end{array}$ & $\begin{array}{l}\text { Only } 30 \text { epochs are } \\
\text { used } \\
\text { More computation } \\
\text { time }\end{array}$ \\
\hline 12 & $\begin{array}{l}\text { Automatic } \\
\text { White Blood } \\
\text { Cell } \\
\text { Detection and } \\
\text { Identification } \\
\text { Using } \\
\text { Convolutional } \\
\text { Neural } \\
\text { Network }\end{array}$ & $\begin{array}{l}\text { Filip Novoselnik, } \\
\text { Ratko Grbić, Irena } \\
\text { Galić, Filip Dorić }\end{array}$ & 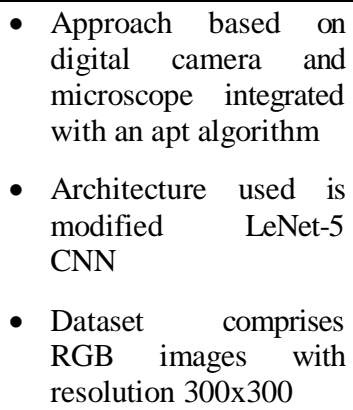 & $\begin{array}{l}\text { In case of WBC } \\
\text { segmentation, the } \\
\text { proposed model attains } \\
\text { a high recall of } 96.66 \% \\
\text { and an accuracy of } \\
90.22 \%\end{array}$ & $\begin{array}{l}\text { - Main limitation of } \\
\text { segmentation method } \\
\text { is that all the } \\
\text { parameters should be } \\
\text { manually allocated to } \\
\text { the suitable values } \\
\text { - Small dataset of } 85 \\
\text { images used }\end{array}$ \\
\hline
\end{tabular}




\section{International Journal of Engineering Technology and Management Sciences[IJETVIS]}

Website: ijetms.in Issue:5, Volume No.4, August-2020

\section{CONCLUSION}

This review paper can be used to compare the results of each paper in the literature survey and draw conclusions based on the methods used in the surveyed papers and their final outcomes. It also gives us an overview of the bigger picture which includes applications of WBCs and how the advancements of different methodologies can be revolutionary in the medical field.

\section{ACKNOWLEDGEMENT}

The authors would like to thank the Department of Electronics and Communication, BMS Institute of Technology, for providing us with an opportunity to present our work and fine-tuning our ideas.

\section{References}

[1] Dhvani Kansara, Shaunak Sompura, Saifil Momin, Mitchell D‘silva, "Classification of WBC for Blood Cancer Diagnosis using Deep Convolutional Neural Networks," International Journal of Research in Advent Technology, Vol.6, No.12, December 2018.

[2] Indraneel Ghosh, Siddhant Kundu Apogee, "Combining Neural Network Models for Blood Cell Classification," BITS Pilani, Pilani,2019.

[3] Muhammed Yildirim, Ahmet Çinar, "Classification of White Blood Cells by Deep Learning Methods for Diagnosing Disease," International Conference, Vol. 33, No. 5, October, 2019, pp. 335340 .

[4] Ming Jiang et al., White Blood Cells Classification with Deep Convolutional Neural Networks, IJPRA.

[5] Nayana B. Sen, Mercy Mathew, "Automated AML Detection from Complete Blood Smear Image Using KNN Classifier," IJAREEIE, Vol. 5, Issue 7, July 2016.

[6] Hüseyin Kutlu, Engin Avci, Fatih Özyurt, "White Blood Cells Detection and Classification Based on Regional Convolutional Neural Networks," Medical Hypotheses (2019).

[7] Poonam Ahire, Tejaswi Gosavi, Shital Bairagi, Pranali More, "Counting Of WBC Using Digital Microscopic Camera in Image Processing," IJREAM, ISSN: 2454-9150 Vol-02, Issue 12, Mar 2017.

[8] Riya roy, Swapna Sasi, "Classification of WBC Using Deep Learning for Diagnosing Diseases," ICICCT 2018.

[9] Merl James Macawile, Vonn Vincent Quiñones, Alejandro Ballado Jr., Jennifer Dela Cruz, Meo Vincent Caya, "White Blood Cell Classification and Counting Using Convolutional Neural Network," in 3rd International Conference on Control and Robotics Engineering, 2018

[10] Mesut Togacar, Burhan Ergen, Mehmet Emre Sertkaya, "Subclass Separation of White Blood Cell Images Using Convolutional Neura Network Models," ELEKTRONIKA IR ELEKTROTECHNIKA, ISSN:1392-1215, VOL. 25, NO. 5, 2019.

[11] Justin L. Wang, Anthony Y. Li, Michelle Huang, Ali K. Ibrahim, Hanqi Zhuang, "Classification of White Blood Cells with PatternNet-fused Ensemble of Convolutional Neural Networks," Conference Paper, December 2018.
[12] Filip Novoselnik, Ratko Grbić, Irena Galić, Filip Dorić, “Automatic White Blood Cell Detection and Identification Using Convolutional Neural Network," International Conference on Smart Systems and Technologies (SST),2018. 